\title{
A influência da irisina na memória em pacientes com doença de Alzheimer: revisão narrativa
}

\author{
The influence of irisin on memory in patients with Alzheimer's disease: narrative review \\ La influencia de la irisina en la memoria en pacientes con enfermedad de Alzheimer: \\ revisión narrativa
}

Kenderly Camila Baltokoski ${ }^{1 *}$, Camila de Melo Accardo.

\begin{abstract}
RESUMO
Objetivo: Revisar a literatura sobre a influência da irisina na memória em pacientes com doença de Alzheimer, através do exercício físico. Revisão Bibliográfica: O exercício físico de resistência promove efeitos positivos na função cognitiva e na plasticidade geral do cérebro, podendo inibir ou retardar o surgimento de doenças neurodegenerativas, como a doença de Alzheimer, através da liberação de mioquinas na corrente sanguínea por meio da atividade endócrina da contração das fibras musculares. A irisina é uma mioquina capaz de proteger o hipocampo, região do cérebro envolvida centralmente no aprendizado e na memória, ao estimular a expressão de fatores neurotróficos, como o fator neurotrófico derivado do cérebro que está envolvido na neuroplasticidade, neurogênese, sobrevivência neuronal, sinaptogênese e cognição. Considerações Finais: Há fortes evidências de que a irisina é capaz de proteger o hipocampo e influenciar na evolução das doenças neurodegenerativas. Contudo, ainda são necessários estudos em maior escala para esclarecer a relação desses fatores na fisiopatologia da doença de Alzheimer e, posteriormente, o possível teor terapêutico.
\end{abstract}

Palavras-chave: Atividade física, Doença de Alzheimer, Irisina, Memória.

\begin{abstract}
Objective: To review the literature on the influence of irisin on memory in patients with Alzheimer's disease, through physical exercise. Literature Review: Resistance physical exercise promotes positive effects on cognitive function and general brain plasticity, and may inhibit or delay the onset of neurodegenerative diseases, such as Alzheimer's disease, through the release of myokines into the bloodstream through endocrine activity contraction of muscle fibers. Irisin is a myokine capable of protecting the hippocampus, a region of the brain centrally involved in learning and memory, by stimulating the expression of neurotrophic factors, such as brain-derived neurotrophic factor, which is involved in neuroplasticity, neurogenesis, neuronal survival, synaptogenesis and cognition. Final Considerations: There is strong evidence that irisin is able to protect the hippocampus and influence the evolution of neurodegenerative diseases. However, larger-scale studies are still needed to clarify the relationship of these factors in the pathophysiology of Alzheimer's disease and, subsequently, the possible therapeutic content.
\end{abstract}

Key words: Physical activity, Alzheimer's disease, Irisin, Memory.

RESUMEN

Objetivo: Revisar la literatura sobre la influencia de la irisina en la memoria en pacientes con enfermedad de Alzheimer, a través del ejercicio físico. Revisión de la literatura: El ejercicio físico de resistencia promueve efectos positivos sobre la función cognitiva y la plasticidad cerebral general, y puede inhibir o retrasar la aparición de enfermedades neurodegenerativas, como la enfermedad de Alzheimer, mediante la liberación de mioquinas en el torrente sanguíneo a través de la actividad endocrina contracción de las fibras musculares.

${ }^{1}$ Centro Universitário das Américas (FAM), São Paulo - SP. “E-mail: kenderly.vda@gmail.com

SUBMETIDO EM: 8/2021

ACEITO EM: 8/2021

PUBLICADO EM: 8/2021 
La irisina es una mioquina capaz de proteger el hipocampo, una región del cerebro que participa centralmente en el aprendizaje y la memoria, estimulando la expresión de factores neurotróficos, como el factor neurotrófico derivado del cerebro, que participa en la neuroplasticidad, neurogénesis, supervivencia neuronal, sinaptogénesis. y cognición. Consideraciones finales: Existe una fuerte evidencia de que la irisina es capaz de proteger el hipocampo e influir en la evolución de las enfermedades neurodegenerativas. Sin embargo, aún se necesitan estudios a mayor escala para esclarecer la relación de estos factores en la fisiopatología de la enfermedad de Alzheimer y, posteriormente, el posible contenido terapéutico.

Palabras clave: Actividad física, Enfermedad de Alzheimer, Irisina, Memoria.

\section{INTRODUÇÃO}

A atividade física é de extrema importância para a promoção da saúde e prevenção de doenças, principalmente crônicas. O exercício, especialmente o exercício de resistência conhecido como aeróbico, pode melhorar a função cognitiva, a plasticidade geral do cérebro e o resultado de doenças neurodegenerativas, sendo que essa melhora tem sido particularmente notada em adultos mais velhos. Desta forma, foi mostrado que o exercício físico melhora resultados relacionados à doença de Parkinson e o Alzheimer, além da depressão, epilepsia e acidente vascular cerebral (DI LIEGRO CM, et al., 2019; ISLAM MR, et al., 2017; YOUNG MF, et al., 2019).

Os benefícios induzidos pela atividade física no cérebro, assim como em outros órgãos, são em parte mediados por peptídeos, como as mioquinas, e metabólitos liberados na corrente sanguínea por meio da atividade endócrina da contração das fibras musculares. No entanto, muitos dos mecanismos moleculares subjacentes ainda não são compreendidos (DI LIEGRO CM, et al., 2019; YOUNG MF, et al., 2019).

As mioquinas regulam uma variedade de processos metabólicos em vários tecidos e órgãos, como fígado, ossos, cérebro ou tecido adiposo por meio de vias de sinalização endócrinas, parácrinas e autócrinas. As principais mioquinas incluem interleucina 6 (IL-6), proteína quimiotática de monócitos 1 (MCP1), fator de crescimento semelhante à insulina-1 (IGF-1) e miostatina (KORTA P, et al., 2019). Em 2012, Boström P, et al. (2012) relatou a descoberta de uma nova mioquina secretada pelos miócitos, chamada de irisina, cuja liberação é induzida pelo exercício e ocorre após a clivagem da fibronectina do tipo III contendo cinco domínios (FNDC5) ligada à membrana (LOURENCO MV, et al., 2019).

A irisina transforma o tecido adiposo branco em tecido adiposo marrom, aumentando assim a termogênese e o consumo de energia do tecido adiposo. No entanto, a contribuição da irisina não se limita à aptidão física e ao escurecimento da gordura, pois também é capaz de proteger o hipocampo ao estimular a expressão de fatores neurotróficos, como o fator neurotrófico derivado do cérebro (BDNF). Como o hipocampo é uma região do cérebro envolvida centralmente no aprendizado e na memória, esse efeito benéfico da irisina pode inibir ou retardar o surgimento de doenças neurodegenerativas, incluindo a doença de Alzheimer. Além disso, os fatores envolvidos na formação da irisina parecem suprimir a agregação de $\beta$-amilóide, que é a marca patológica dessa doença (JIN Y, et al., 2018).

Partindo desta explanação, este trabalho levanta o seguinte problema: a irisina é capaz de melhorar o desempenho da memória em pacientes com doença de Alzheimer, através do exercício físico? Como objetivo geral, o presente trabalho visou revisar a literatura sobre a influência da irisina na memória em pacientes com doença de Alzheimer e como objetivos específicos, estabelecer a relação entre o exercício físico e a saúde do cérebro; elucidar o papel da irisina na doença de Alzheimer; e determinar se a irisina pode atuar como um novo biomarcador sérico de cognição.

\section{REVISÃO BIBLIOGRÁFICA}

Tendo em vista os estudos selecionados nesta revisão, obteve-se algumas informações sobre a temática proposta e, a fim de estabelecer a influência da irisina na memória em pacientes com doença de Alzheimer, 
delimitou-se três categorias: relação entre o exercício físico e a saúde do cérebro; o papel da irisina na doença de Alzheimer; e níveis séricos da irisina e do BDNF e suas atuações como possíveis biomarcadores.

\section{Relação entre o exercício físico e a saúde do cérebro}

Vários estudos evidenciaram que o exercício físico, especialmente o exercício de resistência, promove efeitos positivos diretos e indiretos na saúde do cérebro e na função cognitiva, podendo ser considerado como uma estratégia terapêutica para doenças neurodegenerativas, além de retardar os efeitos do envelhecimento fisiológico (PEDERSEN BK, 2019).

O exercício físico é responsável por uma variedade de efeitos celulares e moleculares, tanto na periferia quanto no cérebro (DI LIEGRO CM, et al., 2019). Há uma alça endócrina entre o músculo e o cérebro, através de peptídeos e metabólitos liberados durante o exercício físico que podem alterar a função do hipocampo diretamente ou estimular a expressão de fatores neurotróficos, como o BDNF (PEDERSEN BK, 2019). Ainda, o exercício aeróbico reverte a perda de volume do hipocampo causando um aumento de $2 \%$, seguido por uma melhora da função de memória, além disso retarda o declínio do funcionamento executivo induzido pela neurodegeneração (JIN Y, et al., 2018).

Nesse sentido, segundo Islam MR, et al. (2017), os efeitos do exercício no cérebro são mais aparentes no hipocampo e no giro denteado, uma parte do cérebro envolvida no aprendizado e na memória. Foi relatado que os efeitos benéficos específicos do exercício no cérebro incluem aumentos no tamanho e no fluxo sanguíneo do hipocampo em humanos, mudanças morfológicas nos dendritos e espinhas dendríticas, aumento da plasticidade das sinapses e, o mais importante, neurogênese de novo no dentado giro em vários modelos de exercícios de camundongos. A neurogênese no cérebro adulto é observada em apenas duas áreas e o giro denteado do hipocampo é uma delas. O exercício é um dos poucos estímulos conhecidos dessa neurogênese de novo (ISLAM MR, et al., 2017). Young MF, et al. (2019), corrobora ao dizer que o hipocampo é uma das áreas cerebrais mais beneficiadas pelo exercício físico, uma vez que ocorre redução da neuroinflamação, além do aumento do fluxo sanguíneo.

O BDNF tem um papel essencial nas melhorias cognitivas induzidas pelo exercício, pois baixos níveis séricos deste fator em humanos têm sido associados a doenças neurodegenerativas, enquanto altos níveis foram associados ao aumento do volume do hipocampo (TARI AR, et al., 2019).

Segundo Morris JK, et al. (2017), o BDNF produzido na periferia é capaz de atravessar a barreira hematoencefálica e desempenhar suas ações centralmente, mas esse fator também é produzido diretamente no cérebro a partir da ação de outras moléculas, algumas, inclusive, produzidas na periferia de maneira dependente do exercício físico. O fator neurotrófico derivado do cérebro está envolvido em vários aspectos importantes no que diz respeito à neuroplasticidade, neurogênese, sobrevivência neuronal, sinaptogênese $e$ cognição (MORRIS JK, et al., 2017).

Ressalta-se que os benefícios observados nos primeiros pacientes com doença de Alzheimer após o exercício aeróbico são devidos ao aumento da aptidão cardiorrespiratória dependente do exercício, que por sua vez está associado ao melhor desempenho da memória e redução da atrofia hipocampal (DI LIEGRO CM, et al., 2019).

Ainda, foi sugerido que os benefícios após o exercício de resistência dependem das condições físicas de cada pessoa, pois foi constatado que as maiores respostas foram obtidas por indivíduos com um melhor condicionamento. Sendo assim, essa evidência destaca que idosos e pacientes com neurodegeneração que apresentam dificuldades para realizar exercícios voluntários podem não obter os resultados almejados (MORRIS JK, et al., 2017).

\section{O papel da irisina na doença de Alzheimer}

A doença de Alzheimer é a forma mais comum de demência relacionada à idade, caracterizada por comprometimento cognitivo, neurodegeneração, deposição de $\beta$-amilóide, formação de emaranhado neurofibrilar e neuroinflamação. Além disso, a neurogênese hipocampal adulta está alterada em pacientes com doença de Alzheimer (CHOI SH, et al., 2018). 
Já a irisina é uma mioquina liberada na corrente sanguínea por meio da atividade endócrina da contração das fibras musculares, capaz de proteger o hipocampo ao estimular a expressão de fatores neurotróficos, como o BDNF, podendo inibir ou retardar o surgimento de doenças neurodegenerativas, incluindo a doença de Alzheimer (JIN Y, et al., 2018).

Além disso, os efeitos protetores da irisina nos neurônios do hipocampo também podem ser atribuídos a uma menor liberação de citocinas inflamatórias pelos astrócitos (DI LIEGRO CM, et al., 2019). Desde sua descoberta em 2012, esta molécula ganhou grande interesse como potencial mediadora dos efeitos promotores da saúde do exercício físico (ZHANG J e ZHANG W, 2016).

A liberação dessa mioquina ocorre após a clivagem da fibronectina do tipo III contendo cinco domínios FNDC5. Durante o exercício, o PGC-1a (coativador 1 alfa do receptor ativado por proliferador do peroxissoma) modula vários fatores secretados pelo músculo esquelético, entre eles, o FNDC5. O exercício aumenta a expressão do FNDC5 no músculo esquelético, levando ao aumento subsequente da irisina circulante, da mesma forma, o exercício de resistência aumenta a expressão do FNDC5 no hipocampo. Ainda, verificou-se que o FNDC5/irisina é altamente expresso nas células de Purkinje do cerebelo, já a irisina foi identificada também no líquido cefalorraquidiano. Além disso, FNDC5/irisina foi detectado em seções hipotalâmicas, especialmente em neurônios paraventriculares (ISLAM MR, et al., 2017; JIN Y, et al., 2018; LOURENCO MV, et al., 2019; YOUNG MF, et al., 2019; ZHANG J e ZHANG W, 2016).

Em relação à doença de Alzheimer, os níveis de FNDC5/irisina são menores no hipocampo e no líquido cefalorraquidiano em pacientes com essa doença neurodegenerativa em estágio avançado quando em comparação aos controles pareados por idade, bem como em camundongos transgênicos (TARI AR, et al., 2019).

Pesquisas recentes destacaram a relação entre a doença de Alzheimer e alterações metabólicas sistêmicas, como desequilíbrio lipídico, hiperglicemia e resistência à insulina, posto que o comprometimento da captação de glicose no cérebro desencadeia atrofia cerebral e disfunção neuronal. Além disso, um estudo realizado em ratos evidenciou que a superexpressão de FNDC5/irisina melhorou a resistência à insulina e reduziu os níveis de glicose no sangue, demonstrando que a irisina pode ter um papel na regulação da homeostase da glicose na doença de Alzheimer (KIM OY e CANÇÃO J, 2018).

Alguns estudos revelaram um papel da neuroinflamação crônica na fisiopatologia de doenças neurodegenerativas como a doença de Alzheimer e a atenção concentrou-se no uso de agentes moduladores anti-fator de necrose tumoral para prevenção e tratamento. Além disso, se provou que os níveis de irisina plasmática e um modelo de camundongo estavam negativamente relacionados com os níveis plasmáticos do fator de necrose tumoral alfa (TNF- $\alpha$ ) e interleucina-6. Neste sentido, evidências sugerem que a irisina pode ser um novo alvo para reduzir mediadores pró-inflamatórios na prevenção ou tratamento desta doença. Além disso, a irisina pode estar mediando efeitos neuroprotetores via astrócitos, diminuindo a liberação de mediadores inflamatórios (DI LIEGRO CM, et al., 2019; JIN Y, et al., 2018; YOUNG MF, et al., 2019).

Dentro da fisiopatologia da doença de Alzheimer, a agregação de $\beta$-amilóide no cérebro pode ser uma das fortes causas da doença, pois aumenta os níveis de espécies reativas de oxigênio e o estresse oxidativo, tornando as células suscetíveis à morte celular por apoptose. As placas de $\beta$-amilóide surgem após a clivagem da proteína precursora amilóide (PPA) presente na membrana celular do neurônio, via enzima BACE-1 (enzima 1 responsável pela clivagem da PPA no local $\beta$ ). No entanto, devido à ativação do coativador de transcrição gênica PGC-1 $\alpha$ após o exercício físico de resistência, não apenas a clivagem da proteína precursora amilóide fica reduzida, mas também a formação de placas de $\beta$-amilóide podem ser prejudicadas, pois o PGC-1 $\alpha$ regula a enzima BACE-1. Além disso, o FNDC5 (precursor da irisina) também pode reduzir a formação de $\beta$-amilóide, pois um estudo recente relatou a interação entre o FNDC5 e a proteína precursora amilóide (JIN Y, et al., 2018).

Outros achados que sugerem que a irisina pode ser um alvo terapêutico em distúrbios neurodegenerativos é o fato de que foi sugerido que a irisina modula a sinalização do transdutor de sinal e ativador de transcrição 3 (STAT3) e provou-se que a proliferação do hipocampo é afetada pelo STAT3. Sendo assim, a síntese da 
irisina após o exercício pode contribuir para diminuir os riscos de doença de Alzheimer, aumentando a proliferação do hipocampo por meio da sinalização STAT3. Esse eixo da irisina pode ampliar a neuroplasticidade, incluindo crescimento, sobrevivência e estabilização neuronal, além de ramificação sináptica (JIN Y, et al., 2018).

Em relação a memória, Choi SH, et al. (2018) afirma que a neurogênese hipocampal adulta induzida pelo exercício melhorou a cognição e a memória, juntamente com a redução da carga de $\beta$-amilóide e com o aumento dos níveis de BDNF, IL-6, FNDC5 e marcadores sinápticos. Segundo esse pesquisador, esses dados sugerem que a promoção da neurogênese pode melhorar a patologia da doença de Alzheimer e os déficits cognitivos, mas apenas na presença de um ambiente cerebral local mais saudável estimulado por exercícios físicos (CHOI SH, et al., 2018).

Em consonância, um ensaio clínico randomizado conduzido por Morris JK, et al. (2017), avaliou o efeito de um programa de seis meses de exercícios aeróbicos supervisionados na memória, na função executiva, na capacidade funcional e na depressão no início da doença de Alzheimer. O estudo evidenciou que o exercício aeróbico está associado a benefícios na capacidade funcional e que os ganhos na aptidão cardiorrespiratória foram associados ao melhor desempenho da memória e à atrofia reduzida do hipocampo, sugerindo que podem ser importantes na promoção dos benefícios cerebrais (MORRIS JK, et al., 2017).

\section{Níveis séricos da irisina e do BDNF e suas atuações como possíveis biomarcadores}

Há muitos desafios para o estabelecimento de um diagnóstico precoce da doença de Alzheimer, justificando a necessidade de novos biomarcadores. Nesse sentido, foi demonstrado que altas concentrações séricas do BDNF amenizam o comprometimento cognitivo. Ademais, dois estudos avaliaram os níveis séricos da irisina e do BDNF, bem como suas atuações como possíveis marcadores biológicos (CONTI E, et al., 2019; KÜSTER OC, et al., 2017).

Kuster OC, et al. (2017), avaliou o fator neurotrófico derivado do cérebro, a irisina e a via da quinurenina (KP) como potenciais novos biomarcadores sanguíneos de cognição, estresse e treinamento físico ou cognitivo em idosos com risco de demência. O estudo evidenciou que, após o exercício ou treinamento cognitivo, os níveis séricos de BDNF e a irisina se correlacionaram positivamente com a cognição global e a memória episódica, enquanto o metabólito neurotóxico quinurenina se correlacionou negativamente com as funções executivas. Isso aumenta a evidência de estudos em animais e in vitro que sugeriram conexões da irisina e seu progenitor FNDC5 com BDNF e neuroplasticidade no sistema nervoso central, como parte da relação entre exercício físico e cognição. Sendo assim, a irisina e a quinurenina podem constituir novos biomarcadores séricos de comprometimento cognitivo, além do já utilizado BDNF (JIN Y, et al., 2018; KÜSTER OC, et al., 2017).

Já o estudo conduzido por Conti E, et al. (2019), que avaliou os níveis séricos de irisina e BDNF, apontou não haver diferenças entre os níveis séricos de irisina em pacientes com doença de Alzheimer versus controles não dementes. Entretanto, a principal descoberta do estudo é que a irisina sérica está significativamente aumentada em pacientes com agitação/agressão nesta doença e se correlaciona com a sua duração. Sendo assim, Conti E, et al. (2019) propôs que essas mioquinas podem representar interessantes resultados em pacientes dementados com sintomas psicológicos e alterações de comportamento que ocasionam atividade motora elevada (CONTI E, et al., 2019).

\section{CONSIDERAÇÕES FINAIS}

A irisina é uma mioquina secretada pelos miócitos, especialmente durante o exercício de resistência. Essa mioquina é capaz de proteger o hipocampo ao estimular a expressão do BDNF, pois esse fator está envolvido na neuroplasticidade, neurogênese, sobrevivência neuronal, sinaptogênese e cognição. Assim, sendo o hipocampo uma região do cérebro envolvida centralmente no aprendizado e na memória, esse efeito benéfico da via FNDC5/irisina-BDNF pode influenciar o resultado de doenças neurodegenerativas, incluindo a doença de Alzheimer. No entanto, embora a irisina seja uma molécula muito interessante e com promessa terapêutica, isso não significa que ela captura todos os benefícios do exercício no cérebro. Por isso, ainda são necessários 
ensaios clínicos em maior escala para esclarecer a relação desses fatores na fisiopatologia da doença de Alzheimer e, posteriormente, o possível teor terapêutico.

\section{REFERÊNCIAS}

1. BOSTRÖM P, et al. A PGC1- $\alpha$ dependentmyokinethatdrivesbrowing of whitefatandthermogenesis. Nature, 2012; 481 : 463-468.

2. $\mathrm{CHOI} \mathrm{SH}$, et al. Combined adult neurogenesis and BDNF mimic exercise effects on cognition in an Alzheimer's mouse model. Science, 2018; 361(6406).

3. CONTI E, et al. Irisin and BDNF serum levels and behavioral disturbances in Alzheimer's disease. Neurological Sciences, 2019; 40(6):1145-1150.

4. DI LIEGRO CM, et al. Physical activity and brain health. Genes, 2019; 10(9): 720.

5. ISLAM MR, et al. The role of FNDC5/Irisin in the nervous system and as a mediator for beneficial effects of exercise on the brain. Springer, 2017; 93-102.

6. JIN Y, et al. Molecular and functional interaction of the myokine irisin with physical exercise and Alzheimer's disease. Molecules, 2018; 23(12): 3229.

7. KIM OY, CANÇÃO J. O papel da irisina na doença de Alzheimer. Journal of Clinical Medicine, 2018; 7(11): 407.

8. KORTA P, et al. Irisin as a multifunctional protein: implications for health and certain diseases. Medicina, 2019; 55(8): 485.

9. KÜSTER OC, et al. Novel blood-based biomarkers of cognition, stress, and physical or cognitive training in older adults at risk of dementia: preliminary evidence for a role of BDNF, irisin, and the kynurenine pathway. Journal of Alzheimer's Disease, 2017; 59(3): 1097-1111.

10. LOURENCO MV, et al. Exercise-linked FNDC5/irisin rescues synaptic plasticity and memory defects in Alzheimer's models. Nature medicine, 2019; 25(1): 165-175.

11. MORRIS JK, et al. Aerobic exercise for Alzheimer's disease: a randomized controlled pilot trial. PloS one, 2017; 12(2): e0170547.

12. PEDERSEN BK. Physical activity and muscle-brain crosstalk. Nature reviews endocrinology, 2019; 15(7): $383-392$.

13. TARI AR, et al. Are the neuroprotective effects of exercise training systemically mediated?. Progress in cardiovascular diseases, 2019; 62(2): 94-101.

14. YOUNG MF, et al. A role for FNDC5/Irisin in the beneficial effects of exercise on the brain and in neurodegenerative diseases. Progress in cardiovascular diseases, 2019; 62(2): 172-178.

15. ZHANG J, ZHANG W. Can irisin be a linker between physical activity and brain function?. Biomolecular concepts, 2016; 7(4): 253-258. 